\title{
Spatial and temporal resonances in a periodically forced hydrodynamic system
}

\author{
F. Marques ${ }^{\mathrm{a}, *}$, J.M. Lopez ${ }^{\mathrm{b}}$ \\ ${ }^{a}$ Departament de Física Aplicada, Universitat Politècnica de Catalunya, Jordi Girona Salgado s/n, \\ Mòdul B4 Campus Nord, 08034 Barcelona, Spain \\ ${ }^{\mathrm{b}}$ Department of Mathematics, Arizona State University, Tempe, AZ 85287-1804, USA \\ Received 5 December 1998; received in revised form 20 May 1999; accepted 10 July 1999 \\ Communicated by S. Fauve
}

\begin{abstract}
The occurrence of a Naimark-Sacker bifurcation in a periodically forced spatially extended system is analyzed with Floquet theory. The basic periodic state of the flow in an annular region consists of the superposition of circular Couette flow, driven by the constant rotation of the inner cylinder, and annular Stokes flow, driven by the harmonic oscillation of the inner cylinder in the axial direction. Windows in parameter space where onset of instability is via Naimark-Sacker correspond to forcing frequencies close to the natural frequencies of the unforced system when the forcing amplitude is sufficiently large. In these windows, not only have strong temporal resonances been identified, but also competition and resonances between various spatial modes that are simultaneously excited take place. An excitation diagram mapping out these windows and loci of resonance points is produced, providing a guide for future explorations into the nonlinear regime by either experimental or computational techniques. (C2000 Elsevier Science B.V. All rights reserved.
\end{abstract}

Keywords: Naimark-Sacker bifurcation; Parametric resonance; Floquet analysis; Taylor-Couette flow

\section{Introduction}

Bifurcation theory is a powerful tool that has experienced extraordinary development over the past few decades. It has supplied the basic building blocks that are very useful in organizing the dynamics of nonlinear systems: bifurcations of fixed points and periodic orbits, homoclinic and heteroclinic connections, and typical chaotic scenarios.

The bifurcations of fixed points of ODE are very well known and simple. The bifurcations of periodic orbits are more complex, but the use of Poincare maps reduces their analysis to the study of fixed points of maps. A limit cycle may lose stability in three generic ways. Two of them are codimension- 1 bifurcations associated with a single real eigenvalue of the Poincaré map crossing the unit circle through either +1 (a saddle-node or a pitchfork bifurcation, depending on the symmetries of the system) or -1 (a period-doubling bifurcation). The third way corresponds to

\footnotetext{
* Corresponding author.
} 
a pair of complex conjugate eigenvalues crossing the unit circle (Naimark-Sacker, a Hopf for limit cycles). The limit cycle with a single frequency bifurcates to a two-dimensional invariant torus with two frequencies. This is a codimension-2 bifurcation; two parameters are necessary in order to unravel all the possible dynamics, which include regions of frequency locking (Arnold's tongues) alternating with quasiperiodic motion, and chaos associated with the presence of strong resonances when the ratio of the two frequencies is a rational number $p / q$, with $q \leq 4$. The overall dynamics in the case of strong resonances is not yet completely understood $[1,11]$.

Parametric resonance is expected when a dynamical system is periodically forced, and the forcing excites some natural frequency of the system. From a dynamical systems point-of-view, the basic state of such a forced system will in general be a limit cycle with the periodicity of the forcing. When some natural frequency of the system becomes excited, a Naimark-Sacker bifurcation results. This bifurcation provides the natural framework for parametric resonance. Unfortunately, a substantial part of the parametric resonance literature has been devoted to Mathieu's and Hill's equations which display a limited range of resonant behavior $(p / q$ with $q=2)$ by comparison with the more general Naimark-Sacker situation. In fact, Mathieu's and Hill's equations are conservative and two-dimensional, and therefore can only undergo either synchronous or subharmonic bifurcations. Moreover, the basic state in these problems is not a limit cycle, but a fixed point.

Naimark-Sacker bifurcations have been reported in many low-dimensional ODE systems, most of them periodically forced, e.g. chemical reactors [9,10] and population dynamics [18]. We are interested in hydrodynamic systems governed by the Navier-Stokes equations (a set of dissipative PDE). When such systems are periodically forced, parametric resonances governed by Naimark-Sacker bifurcations are expected. This has been observed experimentally in periodically forced thermal convection [2,5]. Naimark-Sacker bifurcations have also been observed experimentally and numerically in unforced vortex breakdown flow [20]. The Naimark-Sacker bifurcation is difficult to observe experimentally and to compute numerically as it is codimension-2 and so extensive regions of a two-dimensional parameter space must be explored. When a single parameter is varied, only a one-dimensional slice of the parameter space is observed. One of the most celebrated cases is the so-called quasiperiodic route to turbulence [16,19]. Curry and Yorke [4] showed that this route to turbulence corresponds to a one-dimensional path in the two-dimensional parameter space of a Naimark-Sacker bifurcation.

The Naimark-Sacker bifurcation in low-dimensional dynamical systems exhibits complex behavior associated with temporal resonances. For extended systems, such as in hydrodynamics, various spatial modes can be excited and spatial and temporal resonances between the various modes can be expected to lead to complex behavior.

In this study, we wish to explore spatio-temporal complexity associated with Naimark-Sacker bifurcations in a spatially extended system. We select a temporally forced hydrodynamic system that is readily and precisely reproduced in laboratory experiments and can be simulated numerically. The following section describes this flow in detail, Section 3 discusses competitions between spatial modes and Section 4 deals with the temporal resonances. The results are summarized in a figure mapping out in forcing frequency and amplitude space the regions where various spatial modes occur and the loci of hard temporal resonances as well as loci of spatial resonances and multicritical states.

\section{Description of model problem}

The model system used to explore the onset of Naimark-Sacker bifurcations in spatially extended systems is the flow between two coaxial cylinders, the other one being stationary and the inner one rotating at some fixed rate, with the inner cylinder also subjected to a harmonic oscillation in the axial direction (see Fig. 1). This system has been investigated experimentally $[25]$ and theoretically $[7,12,14]$. The system is governed by a number of non-dimensional parameters. Dimensionally, the inner cylinder oscillates in the axial direction with velocity $U \sin \Omega t$ and rotates 


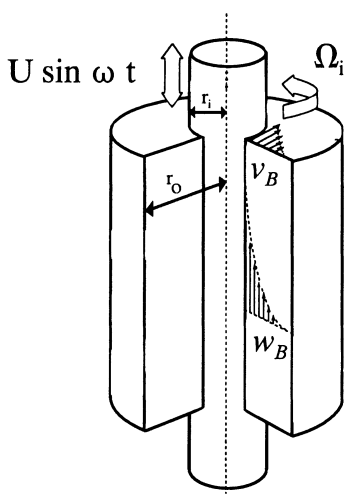

Fig. 1. Schematic of Taylor-Couette flow with axial oscillations of the inner cylinder.

at constant angular velocity $\Omega_{\mathrm{i}}$. Its radius is $r_{\mathrm{i}}$ and the radius of the outer stationary cylinder is $r_{\mathrm{o}}$. The annular gap between the cylinders is $d=r_{\mathrm{o}}-r_{\mathrm{i}}$. These parameters are combined to give the following non-dimensional governing parameters: the radius ratio $e=r_{\mathrm{i}} / r_{\mathrm{o}}$, the Couette flow Reynolds number $R_{\mathrm{i}}=d r_{\mathrm{i}} \Omega_{\mathrm{i}} / \nu$, the axial Reynolds number $R_{\mathrm{a}}=d U / v$, the non-dimensional frequency $\omega=d^{2} \Omega / \nu$, where $v$ is the kinematic viscosity of the fluid. The experimental apparatus [25] has a radius ratio $e=0.905$, and we have used this same value in all the numerical computations presented in this paper.

The basic state consists of the superposition of two basic states, one azimuthal and the other axial. Due to the constant rotation of the inner cylinder, the azimuthal component of velocity is described by circular Couette flow and depends only on the radial coordinate. In the absence of any other mechanism, it loses stability when $R_{\mathrm{i}}$ exceeds a critical value that depends on $e$. Tagg [23] provides a recent guide on the extensive literature for this problem. The axial oscillations of the inner cylinder provide the axial component of the basic state that is the annular analog of Stokes flow ([21], e.g. see [26], p. 321). The annular analog introduces curvature in the azimuthal direction, but the basic state still only has spatial variation in the radial direction and is time periodic. The presence of the outer cylinder becomes very important when the flow is enclosed by endwalls even if the annular gap is large compared to the Stokes layer thickness. The presence of endwalls even if placed far apart, i.e. in the limit of infinite length annulus, produces a back-flow at the outer cylinder in order to conserve mass. This results in an oscillatory boundary layer flow on the outer cylinder similar to the oscillatory Stokes layer on the inner cylinder. These oscillatory boundary layers send waves of azimuthal vorticity, whose sign changes with the stroke of the inner cylinder, into the interior annular region. The endwalls are incorporated into the basic state via a zero net axial mass flux constraint that is enforced by a pressure that is linear in the axial direction. The basic state velocity is

$$
\mathbf{U}_{\mathrm{b}}=\left(0, V_{\mathrm{b}}(r), W_{\mathrm{b}}(r, t)\right)
$$

where

$$
V_{\mathrm{b}}(r)=R_{\mathrm{i}} \frac{r_{\mathrm{i}} r_{\mathrm{o}}}{r_{\mathrm{o}}^{2}-r_{\mathrm{i}}^{2}}\left(\frac{r_{\mathrm{o}}}{r}-\frac{r}{r_{\mathrm{i}}}\right) \quad \text { and } \quad W_{\mathrm{b}}(r, t)=R_{\mathrm{a}} w(r) \sin (\omega t+\alpha(r)) .
$$

$w(r)$ and $\alpha(r)$ are the modulus and phase of $f(r)$ such that

$$
\begin{aligned}
& f^{\prime \prime}+\frac{1}{r} f^{\prime}-\mathrm{i} \omega f=p_{0}, \\
& f\left(r_{\mathrm{i}}\right)=1, \quad f\left(r_{\mathrm{o}}\right)=0 \text { and } \int_{r_{\mathrm{i}}}^{r_{\mathrm{o}}} r f(r) \mathrm{d} r=0 .
\end{aligned}
$$


The integral is the zero net axial mass flux constraint. The function $f(r)$ can be obtained in closed form in terms of modified Bessel functions. The basic state pressure is $p_{1}(r, t)+p_{2}(t) z$, with $p_{2}=0$ if the endwalls are not present. The relationship between $p_{0}$ and $p_{2}$ is given by $p_{2}(t)=R_{\mathrm{a}} \Im\left(p_{0} \exp (\mathrm{i} \omega t)\right)$, and the integral constraint determines the complex constant $p_{0}$.

Although the stability analysis of this basic state can be thought of as a modulated Taylor-Couette problem, particularly when $R_{\mathrm{a}}$ is small or $\omega$ is large, the dynamics of interest in the present study (Naimark-Sacker bifurcations) occur when both components of the basic state (circular Couette and annular Stokes) are comparable. So, one could also view this problem as the centrifugal destabilization of the annular Stokes flow.

The system is governed by the Navier-Stokes equations, which are reduced to a system of ODE by using a Galerkin expansion based on Chebyshev polynomials. The stability analysis of the time-periodic basic state is accomplished by using Floquet theory, and it reduces to the determination of the growth rates of normal mode perturbations from the solution of a linear system of the form:

$$
G \dot{\mathbf{x}}=H(t) \mathbf{x}=(A+B \sin \omega t+C \cos \omega t) \mathbf{x} .
$$

The vector $\mathbf{x}$ contains the coefficients of the perturbations from an expansion of the velocity given by

$$
\mathbf{v}(r, \theta, z, t)=\mathbf{U}_{\mathrm{b}}(r, t)+\exp (\mathrm{i}(n \theta+k z)) \mathbf{u}(r, t) .
$$

The entries in the matrices $G$ and $H$ are given in the appendix of Marques and Lopez [14]. $H$ is periodic, of period $2 \pi / \omega$, where $\omega$ is the frequency of the axial oscillations of the inner cylinder, and $G$ is time-independent and positive definite. The axial and azimuthal wave numbers of the bifurcating solutions are $k$ (real) and $n$ (integer), respectively.

\subsection{Symmetries}

The Navier-Stokes equations governing this problem are invariant under translations $(\tau)$ along and rotations $(R)$ around the common axis of the cylinders. Moreover, there exists an additional discrete symmetry $(S)$ involving time and the axial coordinate; it is a reflection about the plane orthogonal to the axis with a simultaneous time-translation of a half period. Using cylindrical coordinates, they read:

$$
\begin{aligned}
& \left(\tau_{a} \mathbf{v}\right)(r, \theta, z)=\mathbf{v}(r, \theta, z+a), \quad a \in R \bmod 2 \pi / k, \\
& \left(R_{\alpha} \mathbf{v}\right)(r, \theta, z)=\mathbf{v}(r, \theta+\alpha, z), \quad \alpha \in R \bmod 2 \pi, \\
& (S \mathbf{v})(r, \theta, z, t)=\left(v_{r}, v_{\theta},-v_{z}\right)(r, \theta,-z, t+T / 2) .
\end{aligned}
$$

The involutive symmetry $S$ satisfies $S^{2}=I$, and the symmetry group of our problem is $S O(2) \times O(2)$. The presence of these symmetries has many consequences on the dynamics and the bifurcations this system can experience. Chossat and Iooss [3] give details specific to the classical Taylor-Couette problem. In our case, due to the symmetries, we need only consider the $n \geq 0, k \geq 0$ cases.

The presence of the involutive symmetry $S$ prevents period-doubling bifurcations, except in extraordinary cases ([11,22]; see Lemma 7.6, p. 286 of [11]). The main reason is that the Poincare map (the monodromy matrix) is the second iterate of another map. In the present formulation, the symmetry $S$ is used to reduce the region of parameter space to be explored, and the reduced system of equations governing the evolution of perturbations is of the form

$$
\dot{\mathbf{x}}=A(k, t) \mathbf{x}
$$

where $k>0$ and the term $G^{-1}$ in (1) has been included in the right-hand side. The explicit details of the matrices are given in the appendix of Marques and Lopez [14], and these show that $A$ transforms as follows:

$$
A(-k, t+T / 2)=A(k, t) .
$$


The monodromy matrix of (6) is not the second iterate of another map due to the restriction with $k>0$. However, the monodromy matrix of the full system, including positive and negative values of $k$, is the second iterate of a map, as demonstrated in Appendix A. Therefore, period-doubling is not expected in our system according to [22]. We have only observed synchronous bifurcations (pitchforks for limit cycles) and Naimark-Sacker bifurcations with symmetry.

Our basic state, consisting of a superposition of circular Couette flow and annular Stokes flow, is independent of the axial and azimuthal directions, and time-periodic with the period of the forcing. Over an extensive range of parameter space, the primary bifurcation is to an axisymmetric state that is periodic in the axial direction and time, with the same temporal period as the forcing [14,25]. Due to the symmetries of the system, the bifurcation is not the generic fold or saddle-node bifurcation, but a pitchfork for periodic orbits ([11], Theorem 7.12, p. 287). When the basic solution loses stability, two time-periodic solutions resembling Taylor vortices appear; the symmetry $S$ transforms one to the other. The previous works on this problem [7,14,25] focused on delaying the onset of the centrifugal instability of the circular Couette flow by imposing an adequate level of annular Stokes flow, and have considered relatively small amplitude forcing where only synchronous bifurcations resulted. Here, we explore the direct competition between the two mechanisms and how their competition leads to resonance behavior. We locate windows of parameter space where interaction and competition between different axial modes occurs and where the primary bifurcation is to a state that is periodic in both the axial and the azimuthal directions, and temporally has the forcing frequency as well as a new frequency $\omega_{s}$ so that the dynamics are on a torus. We are now in the presence of a Naimark-Sacker bifurcation with symmetry, and these regions in parameter space are pockets of spatio-temporal complexity.

\section{Modal competition}

The experiments and theoretical analysis of the model problem [14,25] clearly demonstrated the effectiveness of the parametric forcing in stabilizing the basic flow and delaying the onset of the centrifugal instability. These works also provided insight into the physical mechanisms involved. In order to gain a quantitative measure of the relative efficiency of the parametric control mechanism, we introduce

$$
\operatorname{Reff}=\frac{\mathcal{N}}{R_{\mathrm{a}}^{2}} \frac{\left(\text { critical } R_{\mathrm{i}}\right)-\left(\text { critical } R_{\mathrm{i}} \text { at zero forcing }\right)}{\text { (critical } R_{\mathrm{i}} \text { at zero forcing) }},
$$

where $\mathcal{N}$ is a normalization factor such that $\operatorname{Reff} \rightarrow 1$ when the forcing is most efficient in delaying the onset of instability in the basic flow to larger $R_{\mathrm{i}}$. This occurs at the double limit $\omega \rightarrow 0$ and $R_{\mathrm{a}} \rightarrow 0$. This ratio gives the degree of enhancement of the critical inner cylinder rotation rate relative to the unforced flow to the amplitude squared of the forcing (the critical $R_{\mathrm{i}}$ at zero forcing is 134.94 for the flow geometry under consideration, i.e. $e=0.905$ ). In fact, $\operatorname{Reff}$ is the ratio between the degree of stabilization obtained and the energy required (proportional to the forcing amplitude squared). Fig. 2 shows Reff as a function of the forcing frequency $\omega$. There is a partial collapse of this relationship (as first noted in [7] at small forcing amplitudes) over a wide range of $R_{\mathrm{a}}$ and particularly for small amplitude and large frequency of the forcing. To quadruple the degree of stabilization at a given frequency, one only needs to double the amplitude.

The dramatic reduction in the efficiency Reff observed in Fig. 2 for small $\omega$ and large $R_{\mathrm{a}}$ comes into play as the non-axisymmetric modes become dominant, excited by the parametric resonance when the forcing frequency is close to the frequency of the spiral modes in the unforced system, and when there is competition between distinct (axisymmetric) modes that simultaneously become critical. The figure gives Reff vs. $\omega$ for six values of $R_{\mathrm{a}}$; from the top curve $R_{\mathrm{a}}=10,30,50,75,100$, and 125 (and $e=0.905$ ). The two dots on the $R_{\mathrm{a}}=125$ curve locate 


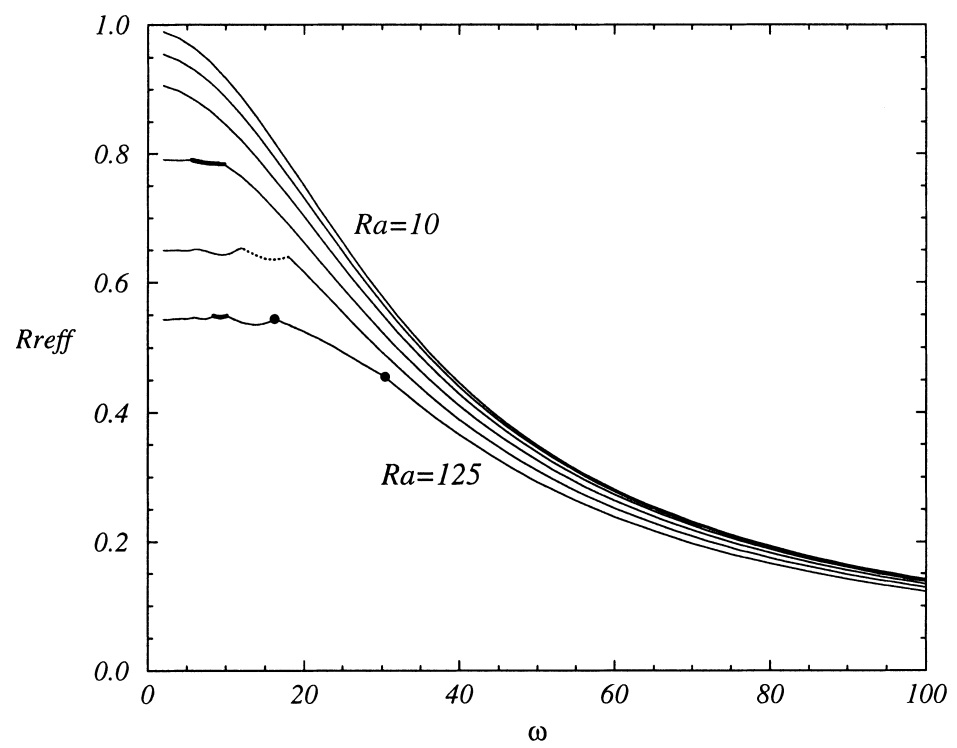

Fig. 2. Relative efficiency $\operatorname{Reff}$ of the forcing in delaying the transition vs. forcing frequency $\omega$ for six values of $R_{\mathrm{a}}$; from the top curve $R_{\mathrm{a}}=10$, $30,50,75,100$, and $125(e=0.905)$. The two dots on the $R_{\mathrm{a}}=125$ curve locate transitions between distinct axisymmetric modes becoming critical. The thick solid line segments correspond to the azimuthal mode $n=1$ being the critical, and the dotted line segment corresponds to the $n=2$ mode. The remaining lines correspond to axisymmetric critical modes.

transitions between distinct axisymmetric modes becoming critical. The thick solid line segments correspond to the azimuthal mode $n=1$ being the critical, and the dotted line segment corresponds to the $n=2$ mode. The remaining lines correspond to axisymmetric critical modes. The curves shown are constructed by taking the absolute minimum in critical $R_{\mathrm{i}}$ over the distinct $n$ for fixed $R_{\mathrm{a}}$ and $\omega$, and plotting them as functions of $\omega$.

The competition between axisymmetric modes is illustrated for the $R_{\mathrm{a}}=125$ case in Fig. 3. The marginal stability curve has several minima and inflection points, and their number varies with $\omega$. For large $\omega(\geq 40)$, the marginal stability curve possesses a single minimum, and locally has the parabolic form typical of the unforced system. When more than one forcing mechanism is present, multiple minima may occur in the marginal stability curves due to competition between the various forces (e.g. $[15,17])$. In our problem, the two competing mechanisms are the centrifugal instability of the circular Couette flow (which results in a cellular structure periodic in $z$ of alternating sign of azimuthal vorticity) and the annular Stokes flow (which sends waves, independent of $z$, of alternating sign of azimuthal vorticity into the annular region from both the inner and outer cylinder walls). As $\omega$ is reduced, an inflection point develops at $k$ greater than the critical value and by $\omega=38.15$, the inflection point has a horizontal tangent. A further decrease in $\omega$ results in a second local minimum at larger $k$, signaling the birth of another solution branch. At $\omega=30.4$, the two local minima have the same $R_{\mathrm{i}}$ value, indicating that eigenvalues corresponding to each of these modes cross the unit circle (at +1$)$ simultaneously. This bi-critical point is marked by a dot in Fig. 2. As $\omega$ is further decreased, the second local minimum at the larger $k$ becomes the dominant mode. At $\omega=18.4$, another inflection point develops a horizontal tangency between the two minima, giving rise to a third branch at lower $\omega$. At $\omega=16.7$, the solution branch that existed for $\omega \geq 40$ merges with an adjacent inflection point and ceases to exist for lower $\omega$. At $\omega=16.42$, the two remaining minima have the same $R_{\mathrm{i}}$ value, and this corresponds to the other dot in Fig. 2. For lower $\omega$, the last formed minimum is dominant, and the two modes are separated in the marginal stability curve by a large maximum between them. Note that the efficiency of the forcing is increasing with decreasing $\omega$ as expected until there is modal competition and switching. Following this, there is a saturation in the efficiency as the critical $R_{\mathrm{i}}$ varies little with further decreases in $\omega$. 
$\omega$.
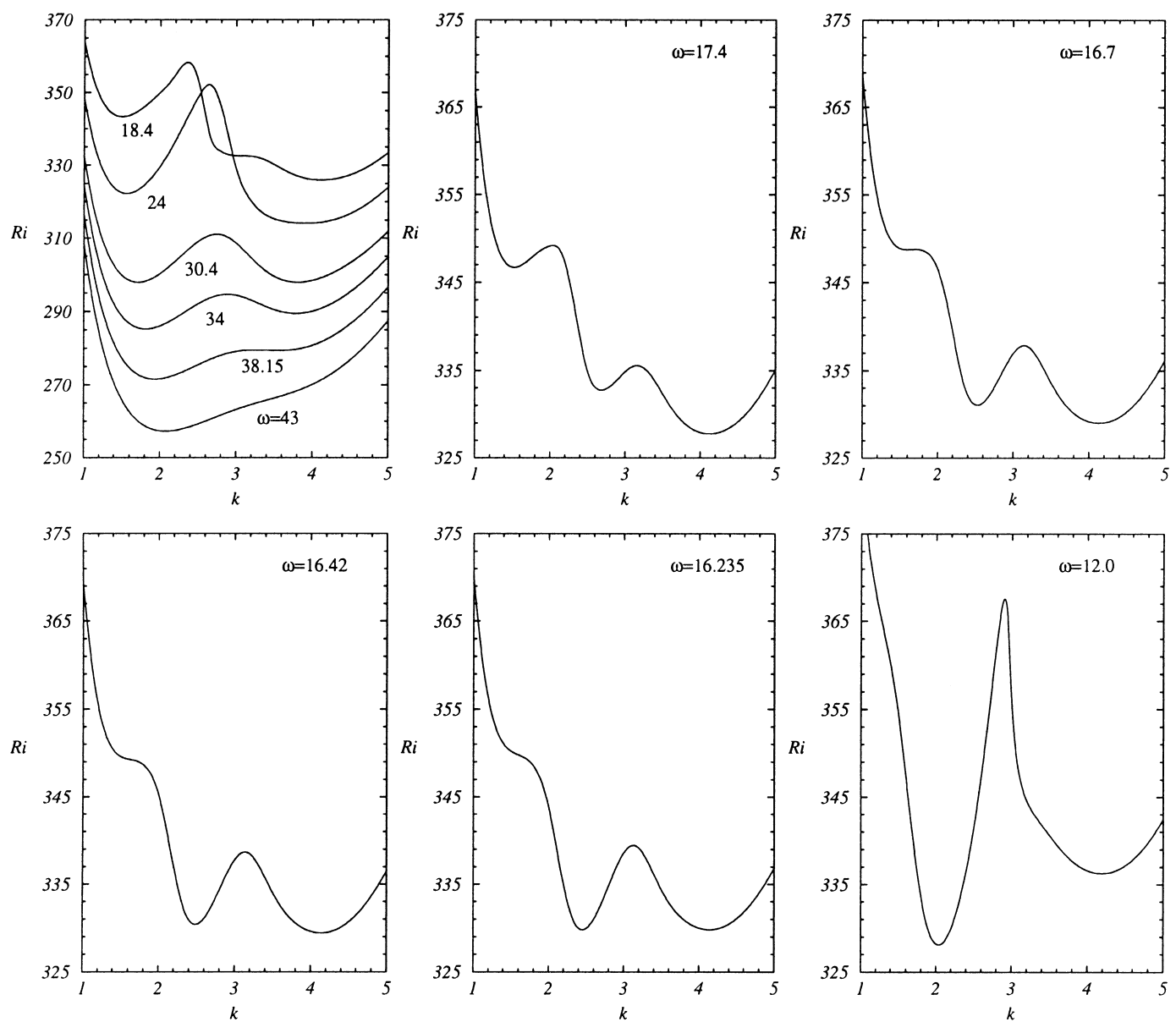

Fig. 3. Marginal stability curves for the azimuthal mode $n=0$ at forcing amplitude $R_{\mathrm{a}}=125$ for various forcing frequencies $\omega$ as indicated (and $e=0.905$ ).

Apart from the competition between distinct axisymmetric modes described above, there is also competition between axisymmetric and non-axisymmetric modes. In the range of parameters where the saturation in Reff is observed, there appear windows where the azimuthal modes $n=1$ and $n=2$ are dominant. For each azimuthal mode $n$, there is also competition between modes with different axial wave numbers $k$. In order to gain a more complete picture of this modal competition, we follow the development of each $(n, k)$ mode for $n=0,1$, and 2 ( $n \geq 3$ do not become dominant for the range of parameters reported here) as the forcing amplitude $R_{\mathrm{a}}$ and frequency $\omega$ are varied. This is summarized in Fig. 4, where the critical values of $R_{\mathrm{i}}$ and $k$ are plotted vs. $\omega$, for various $R_{\mathrm{a}}=65-125$ in steps of 5 . For $R_{\mathrm{a}} \geq 100$, there are more than one local minimum in the marginal stability curves $R_{\mathrm{i}}$ vs. $k$, and these branches are also shown in the figure. The appearance of these multiple solution branches for $n \geq 1$ is analogous to the behavior described above in the $n=0$ case.

The existence of these multiple solution branches is expected to lead to hysteresis in the nonlinear regime close to the bifurcation, with discontinuous jumps in the axial wave number changing by a factor of about 2 as well as discrete jumps in the azimuthal wave number. Further, as the number of modes bifurcating is large, the nonlinear dynamics near the bifurcation is expected to be complicated as they interact and compete. 

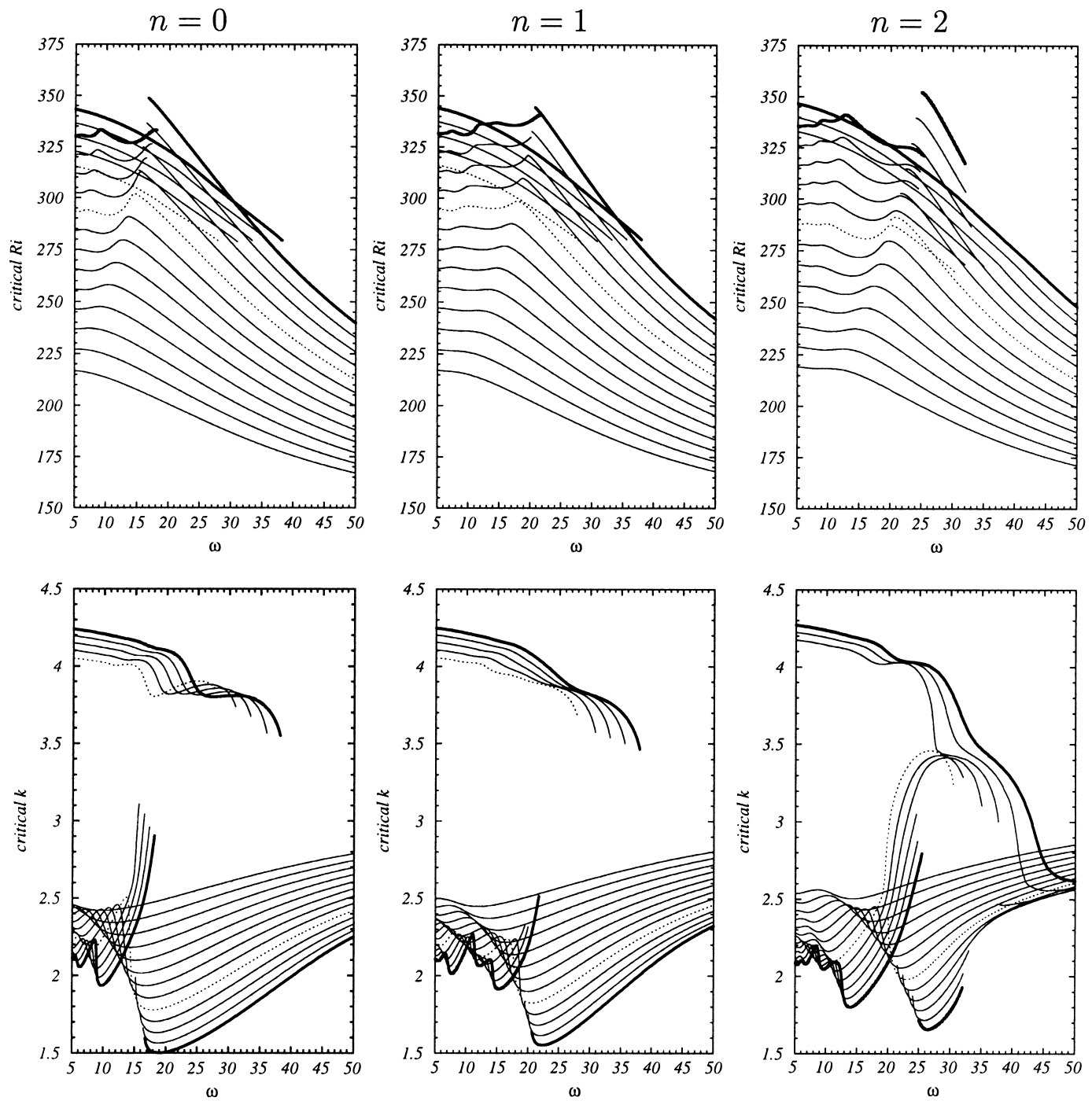

Fig. 4. Critical $R_{\mathrm{i}}$ and $k$ as a function of the forcing frequency $\omega$ for $R_{\mathrm{a}}=65-125$ (in steps of 5), for the azimuthal modes $n=0,1$, and 2, and for all the minima in the marginal stability curves $R_{\mathrm{i}}(k)$, which correspond to the different branches depicted. The branches for $R_{\mathrm{a}}=125$ and all $n$ are thick lines and for $R_{\mathrm{a}}=105$ with $n=0$ and 1 are dashed lines, and for $n=2$ the dashed lines correspond to $R_{\mathrm{a}}=100$. All results are for $e=0.905$.

The forcing not only stabilizes the axisymmetric modes as observed experimentally [25], but it also delays the onset of the non-axisymmetric modes. This is clearly shown in Fig. 4. On average, the degree of stabilization is about the same for all three azimuthal modes (there are specific regions where this is different due to the modal competition as is evident in Fig. 2). In the unforced Taylor-Couette flow, even though the primary bifurcation is to axisymmetric steady Taylor vortex flow, bifurcations also exist to non-axisymmetric time-periodic flows. For example, for an annulus with $e=0.905$ the $n=1$ and $n=2$ modes appear at $R_{\mathrm{i}}=135.59$ and $R_{\mathrm{i}}=137.60$ with natural frequencies 7.111 and 14.45, respectively (the $n=0$ mode is steady and appears at $R_{\mathrm{i}}=134.94$ ). Fig. 4 indicates that the modal interactions begin to occur with increasing $R_{\mathrm{a}}$ for $R_{\mathrm{a}} \geq 68$ and $\omega$ between the natural frequencies of the unforced spiral modes. Although at $R_{\mathrm{a}} \approx 68$ there is only one local minimum in the 
marginal stability curves, the imminent appearance of the other modes at higher $R_{\mathrm{a}}$ begins to be felt, and this shows up as a localized bump that develops into a steep peak in the critical $R_{\mathrm{i}}(\omega)$ curves as well as in the critical $k(\omega)$ curves that at sufficiently large $R_{\mathrm{a}}$ erupts. At this eruption, $\partial$ (critical $\left.k\right) / \partial \omega \rightarrow \infty$. This vertical tangency also occurs when branches terminate as a local minimum merges with an inflection point in the marginal stability curve.

Our observation is that while the axial oscillations of the inner cylinder stabilizes the axisymmetric mode when the amplitude of the oscillations $R_{\mathrm{a}}$ is large enough at frequencies $\omega$ close to the natural frequencies of the unforced system, then it also tends to destabilize the corresponding non-axisymmetric modes. In the geometry considered, for $R_{\mathrm{a}}$ greater than about 68 , we begin to observe primary bifurcations to non-axisymmetric modes.

\section{Temporal resonances}

A distinction between the present system and other dynamical system in which Naimark-Sacker bifurcations have been studied $[9,10,18]$ is that in those other systems their natural frequency at the bifurcation has a fixed known value (by design), and so is independent of the forcing. Following the bifurcation, the two frequencies are known a priori - the imposed forcing frequency and the constant natural frequency. In the present system, the Naimark-Sacker bifurcation leads to a second frequency $\omega_{\mathrm{s}}$, which is analogous to the natural frequency that varies with both the forcing amplitude $R_{\mathrm{a}}$ and frequency $\omega$. It needs to be determined from the Floquet analysis, and a robust technique for this has been presented in [12].

The axisymmetric $n=0$ case in this problem does not undergo a Naimark-Sacker bifurcation; it undergoes a pitchfork bifurcation for limit cycles, and the bifurcating states are synchronous with the forcing. For $n \neq 0$, the system undergoes a Naimark-Sacker bifurcation and introduces a second frequency $\omega_{\mathrm{s}}$. The variation of $\omega_{\mathrm{s}}$ with $\omega$ for $R_{\mathrm{a}}=65-125$, in steps of 5, for the cases $n=1$ and 2 is shown in Fig. 5. These correspond to the solution branches depicted in Fig. 4. In the range of parameters where the mode competition is not prevalent, there is a gradual increase in $\omega_{\mathrm{s}}$ with decreasing $\omega$ and increasing $R_{\mathrm{a}}$. Further, in the limit of $\omega \rightarrow \infty, \omega_{\mathrm{s}}$ tends to the natural frequency of the unforced corresponding spiral mode. Also, independent of $\omega$, in the limit of $R_{\mathrm{a}} \rightarrow 0$, the same limit for $\omega_{\mathrm{s}}$ is obtained. The modal interactions change this behavior dramatically. The eruptions in critical $R_{\mathrm{i}}(\omega)$ and $k(\omega)$ are also observed in $\omega_{\mathrm{s}}(\omega)$. These eruptions are confined to a sector between the lines $\omega_{\mathrm{s}} / \omega=1$ and $\omega_{\mathrm{s}} / \omega=2$ for $n=1$ and $\omega_{\mathrm{s}} / \omega=1$ and $\omega_{\mathrm{s}} / \omega=3$ for $n=2$. Within these sectors, there are several hard resonances $\omega_{\mathrm{s}} / \omega=p / q$ with $q \leq 4$.

We now determine which mode $(n, k)$ is dominant for given $\left(R_{\mathrm{a}}, \omega\right)$, i.e. which mode bifurcates at the lowest $R_{\mathrm{i}}$. This is summarized in Fig. 6, where the critical $R_{\mathrm{i}}(\omega)$ curves are plotted for $R_{\mathrm{a}}=65-125$ in steps of 5. The critical $R_{\mathrm{i}}$, for a given $\left(R_{\mathrm{a}}, \omega\right)$, is the minimum $R_{\mathrm{i}}$ over all $(n, k)$. The curves are plotted as loci with constant $R_{\mathrm{a}}$. The thick lines separate regions corresponding to distinct modes. In region I, $n=1$ dominates and in region II, $n=2$ dominates. Outside regions I and II, $n=0$ dominates, and the two straight thick lines separate distinct $n=0$ modes, with a discontinuous change in $k$ across them. All the thick lines are the loci of multicritical points where multiple modes bifurcate simultaneously. Non-axisymmetric states corresponding to the modes in regions I and II have been observed experimentally [24], but were not studied in detail.

In general, away from the thick lines in Fig. 6 , when $n=0$ there is a pair of real eigenvalues crossing the unit circle at +1 , corresponding to eigenvectors that are related by the discrete symmetry $S$. This corresponds to pitchforks for limit cycles [11]. In regions I and II, where $n \neq 0$, there are two identical pairs of complex conjugate (c.c.) eigenvalues simultaneously crossing the unit circle, again with their respective eigenvectors related by the symmetry $S$. This is a Naimark-Sacker bifurcation with symmetry analogous to the symmetric Hopf for ODE [8], but to our knowledge it has not been studied theoretically. On the thick lines separating different $n=0$ modes, there 

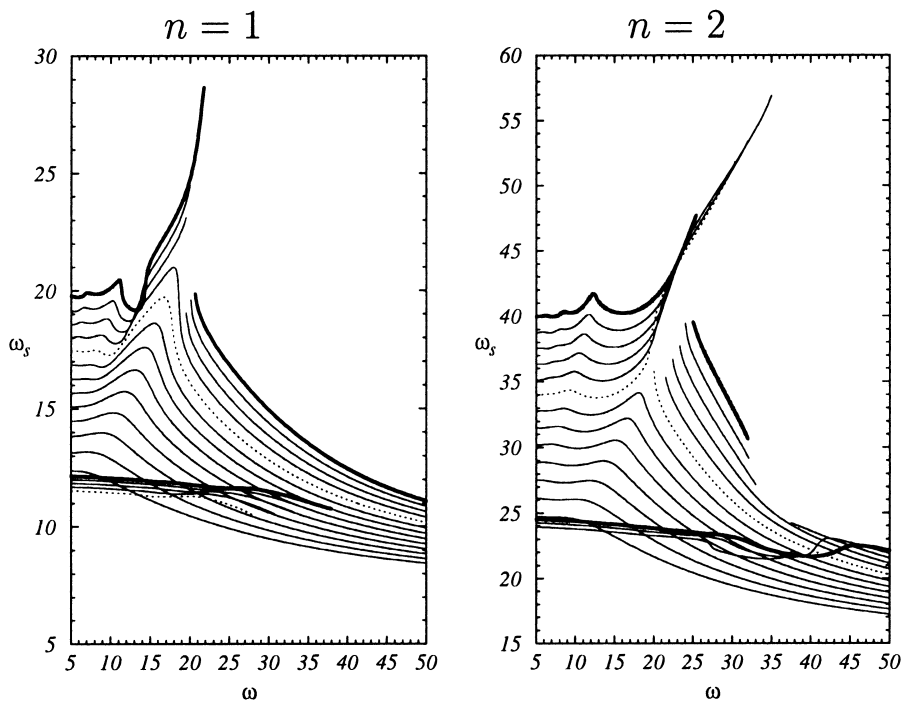

Fig. 5. Second frequency $\omega_{\mathrm{s}}$ as a function of the forcing frequency $\omega$ for $R_{\mathrm{a}}=65-125$ (in steps of 5), for the azimuthal modes $n=1$ and 2 , and for all the minima in the marginal stability curves $R_{\mathrm{i}}(k)$, which correspond to the different branches depicted. The branches for $R_{\mathrm{a}}=125$ and both $n$ are thick lines, for $n=1$ the dashed lines correspond to $R_{\mathrm{a}}=105$ and for $n=2$ they correspond to $R_{\mathrm{a}}=100$. All results are for $e=0.905$.

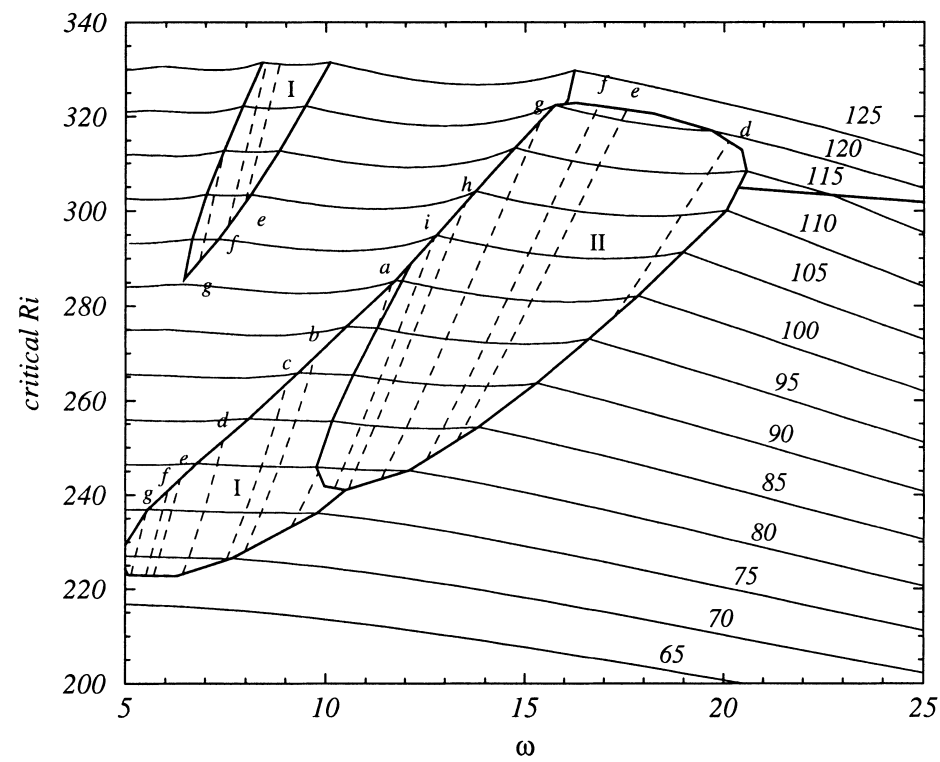

Fig. 6. Critical $R_{\mathrm{i}}$ as a function of the forcing frequency $\omega$ for $R_{\mathrm{a}}=65-125$ as indicated, and $e=0.905$. Thick solid lines separate regions with different dominant modes. Regions I and II have critical azimuthal numbers $n=1$ and 2, respectively. The remaining regions correspond to different $n=0$ modes. Inside the $n \neq 0$ regions, the position of all the strong resonances $p / q$ with $q \leq 4$ are indicated using dashed lines. Labels $a$ to $i$ refer to the strong resonances $3 / 2,5 / 3,7 / 4,2 / 1,9 / 4,7 / 3,5 / 2,8 / 3$ and $11 / 4$. 
are two pairs of real eigenvalues, each pair associated with different values of $k$ that bifurcate. This is a $1: 1$ strong resonance with symmetry. On the thick lines separating either region I or II from the $n=0$ regions, there are a pair of real eigenvalues and two pairs of c.c. eigenvalues bifurcating simultaneously. On the boundary between regions I and II, four pairs of c.c. eigenvalues bifurcate, and more complicated situations arise at the tri-critical points where three regions meet. Apart from the pitchfork for limit cycles, these bifurcations have not been studied theoretically. By analogy with simpler multicritical bifurcations in ODE [6,13], complex dynamics and chaos can be expected following these bifurcations.

Inside regions I and II, there are loci of hard resonance points depicted as dashed lines. These correspond to $\omega_{\mathrm{s}} / \omega=p / q$, with $q \leq 4$, and locate the tips of the widest resonance horns.

\section{Summary}

Theoretical analyses of the Naimark-Sacker bifurcation are usually restricted to asymptotic studies in the limit of either small forcing amplitude or large forcing frequency. In our Floquet stability analysis of a forced extended system as well as in corresponding experimental studies, Naimark-Sacker bifurcations have not been observed in these limits. Windows of Naimark-Sacker bifurcations occur for amplitudes $R_{\mathrm{a}} \geq 65$ and frequencies $\omega \leq 21$. In these windows, the marginal stability curves, $R_{\mathrm{i}}(k)$, for all the azimuthal modes $n$ considered, develop multiple minima corresponding to distinct spatial modes that strongly interact and compete. The windows exist for forcing frequencies in a neighborhood of the natural frequencies associated with non-dominant spiral modes in the unforced system. These modes are excited when the forcing amplitude is sufficiently large and the forcing frequency is resonant with the natural frequencies of the unforced system.

The natural frequencies of the forced system are not known a priori because they depend on the forcing amplitude and frequency (see Fig. 6). These frequencies tend to the known natural frequencies of the unforced system only in the limits $R_{\mathrm{a}} \rightarrow 0$ and $\omega \rightarrow \infty$; and as noted earlier, in these limits the system loses stability via a synchronous pitchfork for limit cycles, and not via a Naimark-Sacker bifurcation.

The extended system considered possesses the symmetry group $S O(2) \times O(2)$ with the $Z_{2}$ symmetry corresponding to a half-period translation in time together with the reflection in $z$. This symmetry prohibits a loss of stability of the basic state via a period-doubling bifurcation. Of course, period-doubling could occur following a symmetry breaking bifurcation.

Nonlinear analysis and experiments are necessary to explore the dynamics beyond the bifurcation points described here and to determine whether the various bifurcations are super or subcritical.

\section{Acknowledgements}

This work was partially supported by DGICYT grant PB97-0685 (Spain), and NSF grants DMS-9706951 and INT-9732637 (USA).

\section{Appendix A. Symmetries and the monodromy matrix}

Let $M_{t}^{\tau}(k)$ be the matrix obtained from (5) by integrating the identity matrix from $t$ to $\tau$. The monodromy matrix of (5) is given by $M_{0}^{T}(k)$ and it obviously satisfies

$$
M_{0}^{T}(k)=M_{T / 2}^{T}(k) M_{0}^{T / 2}(k) .
$$


The symmetry (6) does not apply to (5) since it changes the sign of $k$ and the entries in matrix $A$. In order to study the action of $S$, we consider the enlarged system, including positive and negative values of $k$ :

$$
\left(\begin{array}{l}
\dot{\mathbf{x}} \\
\dot{\mathbf{y}}
\end{array}\right)=\left(\begin{array}{cc}
A(k, t) & 0 \\
0 & A(-k, t)
\end{array}\right)\left(\begin{array}{l}
\mathbf{x} \\
\mathbf{y}
\end{array}\right) .
$$

The solution of this system is given by

$$
\mathbf{x}(t)=M_{0}^{t}(k) \mathbf{x}(0), \quad \mathbf{y}(t)=M_{0}^{t}(-k) \mathbf{y}(0) .
$$

Let us consider now the equation satisfied by $\mathbf{z}(t)=\mathbf{y}(t+T / 2)$,

$$
\dot{\mathbf{z}}(t)=\dot{\mathbf{y}}(t+T / 2)=A(-k, t+T / 2) \mathbf{y}(t+T / 2)=A(k, t) \mathbf{z}(t) .
$$

Therefore,

$$
\begin{aligned}
\mathbf{z}(t) & =M_{0}^{t}(k) \mathbf{z}(0)=\mathbf{y}(t+T / 2)=M_{0}^{t+T / 2}(-k) \mathbf{y}(0)=M_{T / 2}^{t+T / 2}(-k) M_{0}^{T / 2}(-k) \mathbf{y}(0) \\
& =M_{T / 2}^{t+T / 2}(-k) \mathbf{y}(T / 2)=M_{T / 2}^{t+T / 2}(-k) \mathbf{z}(0) .
\end{aligned}
$$

Since $\mathbf{z}(0)$ is arbitrary,

$$
M_{0}^{t}(k)=M_{T / 2}^{t+T / 2}(-k) \Rightarrow M_{0}^{T / 2}(k)=M_{T / 2}^{T}(-k) .
$$

This gives for the monodromy matrix of (5):

$$
M_{0}^{T}(k)=M_{T / 2}^{T}(k) M_{0}^{T / 2}(k)=M_{0}^{T / 2}(-k) M_{0}^{T / 2}(k) .
$$

The monodromy matrix of the enlarged system is

$$
\left(\begin{array}{cc}
M_{0}^{T}(k) & 0 \\
0 & M_{0}^{T}(-k)
\end{array}\right)=\left(\begin{array}{cc}
M_{0}^{T / 2}(-k) M_{0}^{T / 2}(k) & 0 \\
0 & M_{0}^{T / 2}(k) M_{0}^{T / 2}(-k)
\end{array}\right)=\left(\begin{array}{cc}
0 & M_{0}^{T / 2}(-k) \\
M_{0}^{T / 2}(k) & 0
\end{array}\right)^{2} .
$$

Therefore, the monodromy matrix of the enlarged system is the square of a matrix $B$, and the Poincare map is the square of another map. But the matrix $B$ mixes up both components $\mathbf{x}$ and $\mathbf{y}$ corresponding to solutions with $k$ and $-k$. Therefore, the monodromy map of the reduced system (5) will not be a square generically.

\section{References}

[1] V.I. Arnold, Geometrical Methods in the Theory of Ordinary Diiferential Equations, Springer, Berlin, 1988.

[2] A. Chiffaudel, S. Fauve, Strong resonance in forced oscillatory convection, Phys. Rev. A 35 (1987) 4004-4007.

[3] P. Chossat, G. Iooss, The Couette-Taylor Problem, Springer, Berlin, 1994.

[4] J.H. Curry, J.A. Yorke, A transition from Hopf bifurcation to chaos: computer experiments with maps in $R^{2}$, in: J.C. Martin, N.G. Markley, W. Perrizo (Eds.), The Structure of Attractors in Dynamical Systems, Springer Notes in Mathematics, vol. 668, Springer, Berlin, 1978, p. 48.

[5] J.A. Glazier, A. Libchaber, Quasi-periodicity and dynamical systems: an experimentalist's view, IEEE Trans. Circuits and Systems 35 (1988) 790-809.

[6] J. Guckenheimer, P. Holmes, Nonlinear Oscillations, Dynamical Systems, and Bifurcations of Vector Fields, Springer, Berlin, 1986.

[7] H.C. Hu, R.E. Kelly, Effect of a time-periodic axial shear flow upon the onset of Taylor vortices, Phys. Rev. E 51 (1995) $3242-3251$.

[8] G. Iooss, M. Adelmeyer, Topics in Bifurcation Theory and Applications, World Scientific, Singapore, 1992.

[9] T. Kai, K. Tomita, Stroboscopic phase portrait of a forced nonlinear oscillator, Prog. Theoret. Phys. 61 (1979) 54-73. 
[10] I.G. Kevrekidis, L.D. Schmidt, R. Aris, Some common features of periodically forced reacting systems, Chem. Eng. Sci. 41 (1986) $1263-1276$.

[11] Y.A. Kuznetsov, Elements of Applied Bifurcation Theory, 2nd ed., Springer, Berlin, 1998.

[12] J.M. Lopez, F. Marques, Quasiperiodic response to parametric excitations, in: L. Tuckerman, E. Doedel (Eds.), Numerical Methods for Bifurcation Problems and Large Scale Dynamical Systems, IMA Volumes in Mathematics and its Applications, Vol. 119, Springer, Berlin, 1999, pp. 209-228.

[13] A. Mahalov, S. Leibovich, Multiple bifurcation of rotating pipe flow, Theoret. Comput. Fluid Dynamics 3 (1991) 61-77.

[14] F. Marques, J.M. Lopez, Taylor-Couette flow with axial oscillations of the inner cylinder: Floquet analysis of the basic flow, J. Fluid Mech. 348 (1997) 153-175.

[15] A. Meseguer, F. Marques, On the competition between centrifugal and shear instability in spiral Couette flow, J. Fluid Mech. (1999), accepted.

[16] S. Newhouse, D. Ruelle, F. Takens, Occurrence of strange axiom A attractors near quasi-periodic flows on $T^{m}, m \geq 3$, Comm. Math. Phys. 64 (1978) 35.

[17] B.S. Ng, E.R. Turner, On the linear stability of spiral flow between rotating cylinders, Proc. R. Soc. London A 382 (1982) 83-102.

[18] S. Pavlou, I.G. Kevrekidis, Microbial predation in a periodically operated chemostat: a global study of the interaction between natural and externally imposed frequencies, Math. Biosci. 108 (1992) 1-55.

[19] D. Ruelle, F. Takens, On the nature of turbulence, Comm. Math. Phys. 20 (1971) 167.

[20] J.L. Stevens, J.M. Lopez, B.J. Cantwell, Oscillatory flow states in an enclosed cylinder with a rotating endwall, J. Fluid Mech. 389 (1999) $101-118$.

[21] G.G. Stokes, On the effect of the internal friction of fluids on the motion of pendulums, Cambridge Trans. 9 (1851).

[22] J.W. Swift, K. Wiesenfeld, Suppression of period doubling in symmetric systems, Phys. Rev. Lett. 52 (1984) 705-708.

[23] R.P. Tagg, The Couette-Taylor problem, Nonlinear Sci. Today 4 (1994) 1-25.

[24] A.Y. Weisberg, Control of transition in Taylor-Couette flow with axial motion of the inner cylinder, Ph.D. Thesis, Princeton University, 1996.

[25] A.Y. Weisberg, I.G. Kevrekidis, A.J. Smits, Delaying transition in Taylor-Couette flow with axial motion of the inner cylinder, J. Fluid Mech. 348 (1997) 141-151.

[26] C.-S. Yih, Fluid Mechanics, West River Press, Ann Arbour, Michigan, 1977. 